\title{
How Much is at Stake for the Pragmatic Encroacher
}

\author{
Jeffrey Sanford Russell \\ January 2017 draft
}

Many people have said that what you know partly depends on what practical decisions you face. ${ }^{1}$ Charity Anderson and John Hawthorne (this volume) distinguish two different ideas that are typically involved in this "pragmatic encroachment" view. The first idea, which they call "practical adequacy" is that knowledge plays a distinctive role in practical reasoning: you can act on what you know. The second idea is that knowledge is harder to achieve when there is more at stake. It isn't obvious that these two ideas fit together neatly. Indeed, Anderson and Hawthorne convincingly argue that when "high stakes" is made precise in any of several natural ways, situations where more is at stake are often not situations which make stricter demands on practical decisions: so the two ideas come apart. Of course, their discussion leaves some options open: there are other reasonable ways of trying to sharpen the notion of "high stakes", and Anderson and Hawthorne invite others to investigate "whether and to what extent choice of other sharpenings makes a structural difference".

Here I'll extend Anderson and Hawthorne's investigation, and reinforce some of their conclusions, by approaching this question from another direction. My method will not be to try to fit an account of stakes to intuitions about particular cases. Rather, I'll begin by outlining the job that the notion of "high stakes" is supposed to do according to pragmatic encroachers. Then with a bit of decision theory I'll work out what "high stakes" would have to mean in order for it to do this job. What we end up with may or may not be an intuitively plausible account of our ordinary meaning of "high stakes", but even if it isn't, I hope this result will focus the investigation in a productive direction. Since the account of stakes I'll present follows directly from a certain version of pragmatic encroachment together with some standard decision theory, anyone who hopes to advance a rival account of stakes should not just present intuitive cases in support of it, but also explain what alternative theoretical work there is for it to do.

Here's what I'm taking the pragmatic encroacher to say. The first idea is that what you know is certain enough to form a basis for rational action: any proposition you

\footnotetext{
${ }^{1}$ For example, Hawthorne (2003 ch. 4); Stanley (2005); Fantl and McGrath (2009).
} 
know can be rationally taken as given for practical purposes. ${ }^{2}$ In other words, if you know $X$, then you are practically certain of $X$, in the following sense. In a given practical situation, there are certain available actions, the alternative things you are in a position to willingly do.

Definition. In any practical situation, you are practically certain of $X$ iff for every available action $A$, if it is rational to do $A$ conditional on $X$, then it is unconditionally rational to do $A$.

(Anderson and Hawthorne call such a proposition $X$ "practically adequate". For simplicity, in what follows I will identify actions with propositions, such as "I bring an umbrella".)

The second idea is that practical adequacy imposes a certain threshold on the "strength of epistemic position" or "epistemic probability" that you need in order to know $X$ (see for example Fantl and McGrath 2009, 29). I will assume that what we're talking about here is the kind of probability that standardly figures in decision theory, often called your credence in $X$, which will be abbreviated $C(X)$. The thought is that practical adequacy goes along with having high enough credence, where the threshold for how high is high enough depends on what decisions you face.

The third idea is that this practical condition on knowing $X$ goes along with how much is at stake in $X$ being true. In particular, the probability-threshold for practical adequacy is higher in those situations where more is at stake. Putting these three ideas together, we arrive at the following thesis.

High Stakes. For any practical situation $s$ and proposition $X$, there is a threshold $p_{s}(X)$ such that:

(a) Practical certainty means having a credence in $X$ that exceeds this threshold: that is, you are practically certain of $X$ in $s$ iff $C(X) \geq p_{s}(X)$.

(b) For situations $s$ and $t$ and propositions $X$ and $Y$, more is at stake in $X$ in $s$ than in $Y$ in $t$ iff $p_{s}(X)>p_{t}(Y)$.

I will treat this thesis as a constraint on how the pragmatic encroacher understands stakes, and explore what follows from it. (Compare the thesis that Anderson and

\footnotetext{
${ }^{2}$ See Fantl and McGrath (2002); Fantl and McGrath (2007); Hawthorne and Stanley (2008); Hawthorne (2003), p. 178. Note that Fantl and McGrath (2009) no longer endorse this principle.
} 
Hawthorne call "High Stakes". Note that I am supposing that how much is at stake can depend not only on your practical situation, but also on the proposition in question; this corresponds to what Anderson and Hawthorne call "p-stakes".)

I'll also assume a bit of standard decision theory (in the style of Jeffrey 1983). For each proposition $A$, there is a certain quantity which we call your expected value for $A$, abbreviated $V(A)$. The central assumptions are that expected values determine what actions are rational, and that expected values are given by probabilityweighted averages. To be explicit, here are the decision-theoretic principles on which I'll rely.

\section{Decision Theory.}

(a) It is rational to do $A$ unconditionally iff for each available action $B$, $V(A) \geq V(B)$.

(b) It is rational to do $A$ conditional on $X$ iff for each available action $B$, $V(A \wedge X) \geq V(B \wedge X)$.

(c) Expected value is a weighted sum of cases:

$$
V(A)=V(A \wedge X) \cdot C(X \mid A)+V(A \wedge \neg X) \cdot C(\neg X \mid A)
$$

That is, the value of doing $A$ is the weighted average of the value of doing $A$ when $X$ turns out to be true and the value of doing $A$ when $X$ turns out to be false, with each case weighted by how probable it is given that you do $A$.

Finally, I will make some simplifying assumptions about the kind of decision situation that you face. (I'll relax some of these assumptions later.) Suppose you face a forced choice between two actions, $A$ and $B$. Suppose also that the proposition $X$ is simple with respect to your decision: whether $X$ is true is independent of which action you choose, according to your credences. (We aren't concerned here with the more complicated issues involved in knowing things that you are in a position to bring about or prevent. If you are deciding whether to bring an umbrella, then typically "It will rain today" is simple, but "My umbrella will get lost today" is not.) ${ }^{3}$ This means:

$$
\begin{gathered}
C(X \mid A)=C(X \mid B)=C(X) \\
C(\neg X \mid A)=C(\neg X \mid B)=C(\neg X)
\end{gathered}
$$

\footnotetext{
${ }^{3}$ Note that for simplicity I am also setting aside tricky cases in which evidential and causal decision theories give different verdicts: cases in which how you act is good or bad news about how things will turn out in a way that comes apart from its causal impact. In such cases, one might wish to modify Decision Theory to bring in "interventionist" probabilities and expected values, rather than ordinary conditional probabilities (Cartwright 1979, inter alia).
} 
Finally, suppose that $A$ is the only rational action conditional on $X$ : that is,

$$
V(A \wedge X)>V(B \wedge X)
$$

These assumptions straightforwardly imply a particular account of how much is at stake. In this decision situation, acting on $X$ amounts to doing $A$ rather than $B$. So you are practically certain of $X$ iff it is unconditionally rational for you to do $\mathrm{A}$ - that is,

$$
V(A) \geq V(B)
$$

Since expected value is a weighted sum of cases, we can rewrite this:

$$
\begin{gathered}
V(A \wedge X) \cdot C(X \mid A)+V(A \wedge \neg X) \cdot C(\neg X \mid A) \\
\geq \\
V(B \wedge X) \cdot C(X \mid B)+V(B \wedge \neg X) \cdot C(\neg X \mid B)
\end{gathered}
$$

Because $X$ is simple, this can be simplified and rearranged:

$$
(V(A \wedge X)-V(B \wedge X)) \cdot C(X) \geq(V(B \wedge \neg X)-V(A \wedge \neg X)) \cdot C(\neg X)
$$

So, since the left side is positive, it follows that being practically certain of $X$ is equivalent to the following condition:

$$
\frac{C(X)}{C(\neg X)} \geq \frac{V(B \wedge \neg X)-V(A \wedge \neg X)}{V(A \wedge X)-V(B \wedge X)}
$$

The number on the left side of this inequality is your odds for $X$. So in the simple case of just two available actions, you are practically certain of $X$ iff your odds for $X$ are higher than the number on the right side. Let's call the right-hand number the stakes ratio, $S_{S}(X)$ (where $s$ labels a decision scenario of the simple kind we are examining).

Your probability of $X$ is high enough for practical certainty just in case your odds of $X$ are at least $S_{s}(X)$. Since your probability of $X$ gets higher as your odds get higher, this means that the probability-threshold for practical certainty is higher when $S_{s}(X)$ is higher. ${ }^{4}$ Moreover, according to High Stakes there is more at stake exactly when this probability-threshold is higher. So we can conclude that, for two-option situations of the kind under consideration:

${ }^{4}$ Since $C(\neg X)=1-C(X)$, in fact the probability threshold is

$$
p_{s}(X)=\frac{S_{s}(X)}{1+S_{s}(X)}
$$


Stakes Ratio. More is at stake in $X$ in $s$ than in $Y$ in $t$ iff $S_{s}(X)>S_{t}(Y)$

High Stakes and Decision Theory together imply this precise account of relative stakes.

Let's look at what the stakes ratio $S(X)$ is like. (I'll drop the subscript $s$ when it's clear in context which situation we are talking about.) It is a ratio between two quantities, which we can call the cost of being wrong about $X, V(B \wedge \neg X)-V(A \wedge \neg X)$, and the benefit of being right about $X, V(A \wedge X)-V(B \wedge X)$. Recall that, by assumption, $A$ is the best action conditional on $X$; so if you rationally act on $X$, then $A$ is what you do. So $V(A \wedge \neg X)$ is the expected value of acting on $X$ when $X$ turns out to be false. The cost of being wrong about $X$ is how much worse acting on the mistaken assumption of $X$ is than the alternative action would be. As this cost gets bigger, more is at stake in $X$ being true. The benefit of being right about $X$ is how much better it is to act on $X$ than its alternative, supposing $X$ turns out to be true. As this benefit increases, the stakes ratio goes down: since there is more in favour of acting on $X$, it takes less confidence to make it rational to do so. ${ }^{5}$

As an example, consider the classic "bank case" (DeRose 1992). First version. It's Friday afternoon, you need to deposit your paycheck, and there are long lines at the bank. The bank might be open Saturday, and if it is, the lines will be much shorter. If you wait till Saturday and it turns out to be closed, that would be kind of a nuisance.

\begin{tabular}{lcc}
\hline Actions & Bank open Saturday & Bank closed Saturday \\
\hline Go Saturday & Short wait & Nuisance \\
Go Friday & Long wait & Long wait \\
\hline
\end{tabular}

\footnotetext{
${ }^{5}$ This account of stakes is in a similar spirit to a proposal that Anderson and Hawthorne briefly discuss in Section 3:

Here one natural idea for calculating $p$-stakes is to compare the expected utility of an action conditional on $p$ and the expected utility of that action conditional on not- $p$. ... On this gloss, the larger the gap between these conditional expected utilities the more is at stake for that action when it comes to $p . "$ (PAGE REF).

They go on to refine this idea in terms of regret; this refinement brings their proposal even closer to Stakes Ratio, since it also takes into account the conditional expected values for those actions not taken. (One important way Stakes Ratio is different from this is that it uses a ratio rather than an absolute difference; thus the stakes for $X$ and $\neg X$ are not equal, but rather inverse.) What we have shown is that a specific implementation of this general kind of proposal is a consequence of High Stakes and Decision Theory.
} 
In this case the stakes ratio is

$$
S(\text { Bank open Saturday })=\frac{\text { Long wait }- \text { Nuisance }}{\text { Short wait }- \text { Long wait }}
$$

Second version: same as before, except if you don't make the deposit on Friday or Saturday then an important check will bounce, which would be really terrible.

\begin{tabular}{lcc}
\hline Actions & Bank open Saturday & Bank closed Saturday \\
\hline Go Saturday & Short wait & Terrible things \\
Go Friday & Long wait & Long wait \\
\hline
\end{tabular}

$$
S(\text { Bank open Saturday })=\frac{\text { Long wait }- \text { Terrible things }}{\text { Short wait }- \text { Long wait }}
$$

In this version, there is a much bigger cost of being wrong about whether the bank is open Saturday: there is a much bigger gap in value between a long wait and the terrible things that may ensue than there is between the long wait and the minor nuisance. So there is more at stake in the bank being open in the second situation. (This is what you might hope, since "Low Stakes" and "High Stakes" are standard labels for these two cases).

Note that in situations where which action is best doesn't turn on $X$ at all - the case where $V(B \wedge \neg X)$ isn't any better than $V(A \wedge \neg X)$ - then the stakes ratio isn't even positive. In that case practical adequacy doesn't impose any constraint on your probabilities at all.

We can generalize Stakes Ratio to cases where more than two actions are available with only a slight modification. If $A$ is the uniquely best action conditional on $X$, then practical certainty requires that $V(A)$ be unconditionally at least as good as each available alternative. So by parallel reasoning, we can derive in this more general case:

$$
S_{S}(X)=\max _{B \neq A} \frac{V(B \wedge \neg X)-V(A \wedge \neg X)}{V(A \wedge X)-V(B \wedge X)}
$$

(This calculation is still based on the assumption that $X$ is simple with respect to your available actions. $)^{6}$

\footnotetext{
${ }^{6}$ The case where different actions $A$ and $A^{\prime}$ are exactly tied for best, conditional on $X$, is slightly messier. In this case, if $V(A \wedge \neg X) \neq V\left(A^{\prime} \wedge \neg X\right)$, then the stakes ratio is infinite. Only certainty that $X$ is true is enough guarantee that both of these conditionally rational actions are also unconditionally
} 
The stakes ratio has a peculiar feature: it isn't sensitive to the absolute magnitudes of the costs and benefits involved, just their proportions. For example ${ }^{7}$ suppose I have to choose between two boxes labeled $\mathrm{A}$ and $\mathrm{B}$, exactly one of which contains $\mathcal{E}$. And suppose you have to choose between two other boxes also labeled $\mathrm{A}$ and $\mathrm{B}$, exactly one of which contains $£ 1000$.

\begin{tabular}{lcc}
\hline Actions & Money in Box A & Money in Box B \\
\hline Box A & $\mathcal{E}^{1}$ & 0 \\
Box B & 0 & $\mathcal{E}^{1}$ \\
\hline
\end{tabular}

\begin{tabular}{lcc}
\hline Actions & Money in Box A & Money in Box B \\
\hline Box A & $£ 1000$ & 0 \\
Box B & 0 & $£ 1000$ \\
\hline
\end{tabular}

In each of our two situations, the stakes ratio for the money being in Box $\mathrm{A}$ is $1: 1$, since the cost of wrongly acting on the assumption that the money is in Box A is just the same as the benefit of rightly acting on that assumption. So we get the conclusion that there is exactly as much at stake in the money being in Box A in my $£ 1$ situation as there is in your $£ 1000$ situation. In each case, the probability that makes it rational to choose one box over the other is just $\frac{1}{2}$. So what it takes to make it rational to act on the money being in Box $\mathrm{A}$ is equally undemanding for each of us (assuming that choosing a box is the only relevant action). But the corresponding judgment about how much is at stake this is at least a bit counter-intuitive. It seems more natural to say that there is more at stake in the money being in Box A when there is more money involved.

But remember, we didn't get this far by trying to respect intuitions about cases. Rather, we derived consequences from certain pragmatic encroachment theses together with decision theory. So we face a choice. Give up standard decision theory. Accept an account of how much is at stake which is at odds with some of our intuitive responses to cases like these. Or give up the idea that how much is at stake is what sets the threshold for a practical condition on knowledge, either by giving up

rational. Otherwise, suppose there are multiple tied best actions conditional on $X$, and all of these actions are also tied conditional on $\neg X$. In that case, we can let $A$ be any one of them and use the same formula for $S_{s}(X)$ given above, with the only modification being that the variable $B$ should range just over those actions which are not among the tied best actions conditional on $X$ - that is, those actions $B$ such that $V(A \wedge X)>V(B \wedge X)$.

${ }^{7}$ Thanks to Charity Anderson for helpful discussion of this case. 
pragmatic encroachment altogether or by finding some other way of characterising the view. ${ }^{8}$

\section{References}

Anderson, Charity, and John Hawthorne. this volume. "Knowledge, Practical Adequacy, and Stakes."

Cartwright, Nancy. 1979. "Causal Laws and Effective Strategies." Noûs 13 (4): 419-37. http://www. jstor.org/stable/2215337.

DeRose, Keith. 1992. "Contextualism and Knowledge Attributions." Philosophy and Phenomenological Research 52 (4): 913-29. http://www.jstor.org/stable/ 2107917.

Fantl, Jeremy, and Matthew McGrath. 2002. "Evidence, Pragmatics, and Justification." Philosophical Review 111 (1): 67-94. http://dx.doi.org/10.1215/ 00318108-111-1-67.

- 2007. "On Pragmatic Encroachment in Epistemology." Philosophy and Phenomenological Research 75 (3): 558-89. http://dx.doi.org/10.1111/j. 1933-1592.2007.00093.x.

. 2009. Knowledge in an Uncertain World. Oxford University Press. http: //dx.doi.org/10.1093/acprof : oso/9780199550623.001.0001.

Hawthorne, John. 2003. Knowledge and Lotteries. Oxford University Press. http: //dx.doi.org/10.1093/0199269556.001.0001.

Hawthorne, John, and Jason Stanley. 2008. "Knowledge and Action." Fournal of Philosophy 105 (10): 571-90. http://www.journalof philosophy.org/ articles/issues/105/10/1.pdf.

Jeffrey, Richard. 1983. The Logic of Decision. University of Chicago Press.

Stanley, Jason. 2005. Knowledge and Practical Interests. Oxford: Oxford University Press. http://dx.doi.org/10.1093/0199288038.001.0001.

\footnotetext{
${ }^{8}$ Thanks to Charity Anderson, Julien Dutant, Daniel Eaton, John Hawthorne, Tim Pickavance, and the other participants at the workshop on Religious Epistemology, Contextualism, and Pragmatic Encroachment at Oxford in March 2013. This project was made possible through the support of a grant from the John Templeton Foundation. The opinions expressed in this publication are those of the author and do not necessarily reflect the views of the John Templeton Foundation.
} 